\title{
SPRITE: the Spitzer proposal review website
}

\author{
Megan K. Crane, Lisa J. Storrie-Lombardi, Nancy A. Silbermann, Luisa M. Rebull \\ Spitzer Science Center, California Institute of Technology, MS 220-6 \\ 1200 E. California Blvd., Pasadena, CA, USA 91125
}

\begin{abstract}
The Spitzer Science Center (SSC), located on the campus of the California Institute of Technology, supports the science operations of NASA's infrared Spitzer Space Telescope. The SSC issues an annual Call for Proposals inviting investigators worldwide to submit Spitzer Space Telescope proposals. The Spitzer Proposal Review Website (SPRITE) is a MySQL/PHP web database application designed to support the SSC proposal review process. Review panel members use the software to view, grade, and write comments about the proposals, and SSC support team members monitor the grading and ranking process and ultimately generate a ranked list of all the proposals. The software is also used to generate, edit, and email award letters to the proposers. This work was performed at the California Institute of Technology under contract to the National Aeronautics and Space Administration.
\end{abstract}

Keywords: Spitzer Space Telescope, Proposal Peer Review, Telescope Time Allocation, Web Database Application, MySQL, PHP

\section{INTRODUCTION}

The Spitzer Proposal Review Website (SPRITE) is a PHP/MySQL web database application designed to support the Spitzer proposal review and Time Allocation Committee (TAC) process. A web database application is a web site that is driven from data stored in a database. The application is generally built around a three-tier architecture model. At the base is the database tier, which contains the data that users may create, delete, modify, or query. The middle tier is the application logic, which consists of the webserver and the scripts that communicate the data between the other two tiers. The top tier is the client web browser software.

This application was developed on Mac OS X using the popular open source trio of the PHP server-side scripting language, the MySQL database management system, and the Apache webserver. The application utilizes the Secure Sockets Layer (SSL) protocol, which provides encryption services to ensure secure web transactions. PHP was chosen for its power, flexibility, platform and browser independence, compatibility with MySQL, and its native session and cookie management, which are used to control user access. MySQL is a high-performance backend database management system with a high-speed transactional processing system that can support over a hundred simultaneous users of the software.

\subsection{System Requirements}

1. A webserver such as Apache that can execute PHP scripts

2. PHP version 4.x or 5.x

3. MySQL version 4.x or 5.x

4. Postfix or sendmail to send emails via PHP scripts

5. FPDF - a free PHP class used to generate PDF files

\subsection{Load Test}

A load test of the SPRITE software was conducted using SilkPerformer $6.6^{1}$. The test consisted of 120 users overloading the system: 11 review panels with 10 different reviewers on each panel simultaneously entering grades on 800 different proposals, and 10 SSC support team members each monitoring their panel's grades, entering allocations and notes, and simultaneously calculating the average grade of all of the proposals in their panel. Response times slowed considerably, but no system degradation was observed and no errors logged. The SPRITE software behaved beautifully with no dropped connections, stuck threads, or time-outs. No major performance bottlenecks were predicted during operational use.

Observatory Operations: Strategies, Processes, and Systems II, edited by Roger J. Brissenden, David R. Silva, Proc. of SPIE Vol. 7016, 701625, (2008) $\cdot 0277-786$ X/08/\$18 $\cdot$ doi: $10.1117 / 12.788559$ 


\subsection{Motivation for SPRITE}

The former Spitzer proposal review process has been described in detail in Laine et al. ${ }^{2}$. The voting process used to involve reviewers voting by secret ballot, with SSC support team members collecting the ballots by hand and entering the votes into the old review software. The desire for reviewers to be able enter their grades electronically drove the development of SPRITE.

The old review software consisted of cgi scripts and Perl hash database tables. Once the tables were created, it was very difficult to modify them. A more flexible system was needed that could easily handle updates to conflicts and proposal assignments, since these would change frequently as reviewers began to read the proposals.

In the past, award and rejection emails were sent to the proposers by hand. Since this involved sending 700-800 emails one at a time to over 5000 recipients, it was desired to design a system that could generate and send the letters automatically, and include the proposal's quartile and the reviewers' comments.

\subsection{Summary of the proposal review process}

1. Reviewers enter preliminary grades and comments about the proposals.

2. The proposals with the lowest preliminary average grade are triaged.

3. Reviewers meet in person in Pasadena for a week to discuss and re-vote on the non-triaged proposals, generate a ranked list of all of the proposals, and write final comments.

4. Award letters are emailed to the proposers that include the comments written by the reviewers.

\section{MYSQL DATABASE SCHEMA \& BACKUPS}

The SPRITE database contains information about all of the proposals and all of the users of the system, including each reviewer's individual grade and comments for each proposal and the average grade for each proposal. There are eight tables in the database: users, pids, grades, grades_tac, averages, comments_final, panel_alloc, and emails.

\subsection{The users table}

The users table contains the full name, username, password, panel assignment, and status designation for each user. There are typically about 100 review panel members who are assigned to one of 11 science panels. The SSC Science User Support Team (SUST) members are assigned to one of the 11 panels to monitor the voting and ranking process for that panel. The TAC chair and SSC Community Affairs Team (CAT) members are not assigned to a specific panel. There are seven different panel categories in the users table:

1. $1 A, 1 B, 1 C$ - Extragalactic science panels for reviewers and SUST members (intermediate/high-z galaxies, cosmology, GRBs, AGN/QSOs, ULIRGs, high-z clusters)

2. $2 A, 2 B$ - Extragalactic science panels for reviewers and SUST members (local group, nearby galaxies, low$\mathrm{z}$ clusters, interacting/merging galaxies)

3. $3 A, 3 B, 3 C$ - Galactic science panels for reviewers and SUST members (ISM, star formation, young stellar objects, galactic structure, HII regions, massive stars, evolved stars, extragalactic stellar studies, clusters)

4. $4 A, 4 B$ - Galactic science panels for reviewers and SUST members (circumstellar disks, brown dwarfs, extrasolar planets)

5. 5-Solar system panel for reviewers and one SUST member

6. $t a c-$ for TAC chair

7. cat-for CAT members

There are eight different status designations in the users table, which are used to control user access:

1. rev-reviewers

2. chair - panel chairs who also comprise the TAC

3. dep - deputy panel chairs

4. sust - the SSC Science User Support Team

5. cat - the SSC Community Affairs Team

6. $t a c$ - the TAC chair

7. pun - pundits who participate in the TAC but are not on panels

8. dir- members of the SSC Director's Office 
Passwords in the users table are encrypted using the MD5 algorithm in conjunction with a unique salt, a string of extra characters prepended and appended to the password.

\subsection{The pids table}

The pids table contains information about each proposal. Each proposal is assigned to one panel depending on its science category. Some Medium ( $>50$ hours) and Large ( $>200$ hours) proposals are assigned to two parallel panels.

Information in the pids table consists of the proposal title, Principal Investigator (PI) information, requested/allocated hours (for General Observer, Guaranteed Time Observer, and Legacy proposals) or dollars (Archival Research and Theoretical Research proposals), technical notes about the proposals including Target of Opportunity and observing constraint information, primary and secondary reviewers, reviewers conflicted with the proposal, status values (Triage, Accept, or Reject), quartile, and scheduling priority. The information in the pids table must be extracted from the SSC proposals database and loaded into the SPRITE database prior to the proposal review meeting.

\subsection{The grades table}

The grades table holds the individual grades, votes, and preliminary comments for each reviewer for each proposal. There are two rounds of grading: preliminary grading, which happens over a period of several weeks before the review meeting, and voting which happens during the week-long review meeting in Pasadena. The preliminary grades are used to guide the triage process (see section 3.4.2). The preliminary comments entered by each reviewer are combined into one field per proposal just prior to the review meeting. The combined comments are transferred to the comments_final table, which can then be edited by the reviewers during the review meeting.

\subsection{The grades_tac table}

The grades_tac table holds the grades for the panel chairs and the TAC chair for each proposal in the TAC (i.e. the Medium and Large proposals).

\subsection{The averages table}

The averages table holds the average, standard deviation, $\mathrm{N}$ (number of grades), minimum, and maximum grade for each proposal for each round of grading (preliminary, voting, and TAC voting). The preliminary average is calculated automatically by SPRITE as reviewers enter their grades, but the voted averages must be calculated explicitly by the SSC support team members during the review meeting (see section 3.3.1). The averages table also holds the rank of each proposal as determined in the panels as well as the TAC.

\subsection{The comments_final table}

The comments_final table holds the final comments for each proposal. The primary reviewer is responsible for editing the final version of the comments, though each reviewer in the panel has the ability to edit them.

The comments are overwritten in the database each time any reviewer updates them. However, every version of the comments is stored in a file on the hard disk in case it is necessary to refer back to an older version.

\subsection{The panel_alloc table}

The panel_alloc table holds the provisional allocation for each panel, which is the number of hours to be allocated to the Small proposals in the panel. The TAC also has a provisional allocation, which is the number of hours to be allocated to the Medium and Large proposals.

\subsection{The emails table}

The emails table holds the email addresses of the Principal Investigator and all of the co-investigators for each proposal. These are used to send the award letters after the review meeting (see section 3.4.4). The email addresses must be extracted from the SSC proposals database and loaded into the SPRITE database.

\subsection{Database backups}

MySQL has a built-in utility for backing up databases called mysqldump, which generates a text file of SQL commands needed to rebuild the database and all of the tables from scratch. There are two dedicated webservers for SPRITE; one hosts the application and the other is used for backups. In the weeks leading up to the review meeting, while reviewers are entering their preliminary grades, the database is backed up once a day. The mysqldump command is run via a cron 
job, and the resulting output file is transferred (using ssh) to the backup webserver. The file is loaded into a database on the backup webserver so there will be an exact copy of the database on each webserver. During the review meeting, the database is backed up every 15 minutes, which does not affect the performance of the application in any way.

Likewise, all of the application PHP scripts, HTML and CSS files, and other related files (images, proposal PDFs, comments files, etc.) are copied over to the backup server daily using cron, ssh, and rsync so that there will be an exact copy of the application on both servers.

\section{THE PHP WEB APPLICATION}

\subsection{Logins and logouts}

When users login to SPRITE (see Figure 1), they enter a username and password into a web form. The password is encrypted using the MD5 algorithm and a salt specific to the SPRITE application. If the entered password matches the password in the database for that username, login is successful. If not, the user is redirected to the login page with an error message stating that the login was unsuccessful.

Upon successful login, the users table is queried for other information about the user such as his/her status designation and the panel to which he/she is assigned. This information is registered in PHP session variables, stored in the PHP superglobal array $\$ \_S E S S I O N$, and stored in a cookie in the user's browser. This means that users must configure their browser to accept cookies. The session information is maintained as long as the user is logged in. Depending on their status, users are allowed access only to certain pages. For example, only the panel chairs are allowed to view the individual grades of each reviewer in their panel, and SUST members are only allowed to monitor the voting and ranking of the panel to which they are assigned. Error messages are displayed and logged if a user tries to access an unauthorized page.

When a user logs out of SPRITE, their session information is destroyed and they are redirected back to the login page. They cannot access any pages without logging in again.

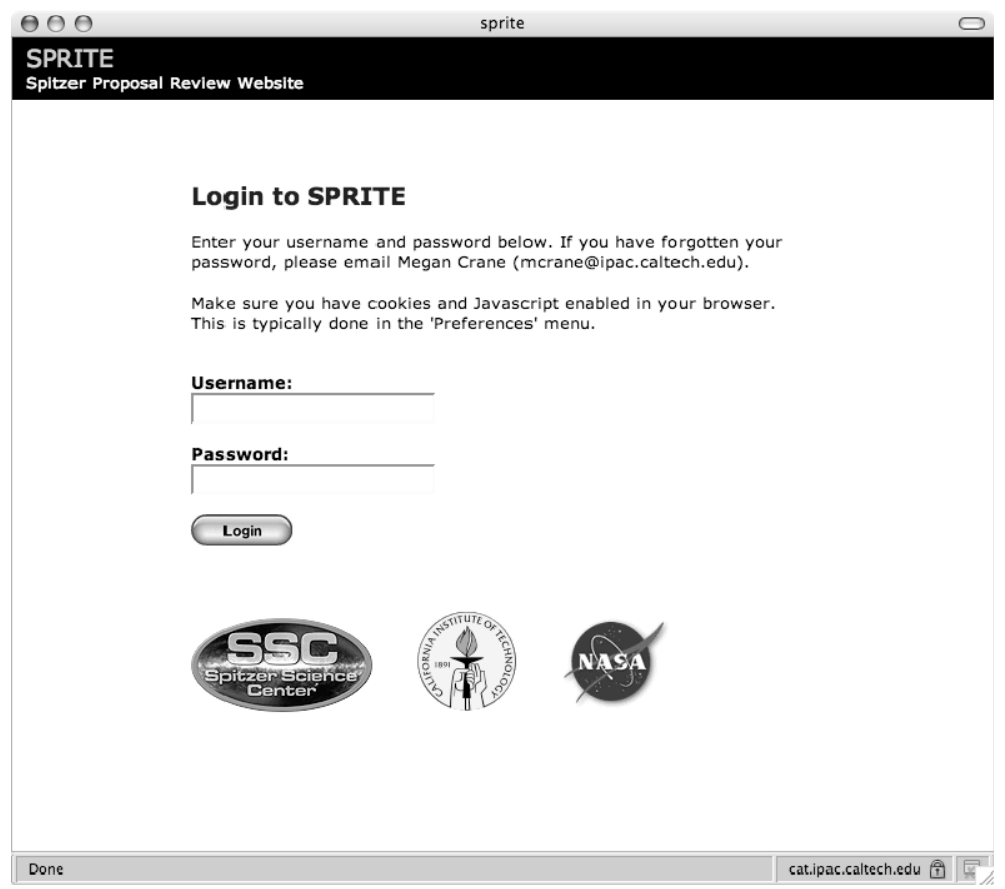

Fig. 1. The SPRITE login page. All users of the software use this page to login, and they are presented different options once they login depending on their status designation.

\subsection{SPRITE options for reviewers/panel chairs}

Reviewers read, grade, rank, and write comments about the proposals; they are grouped into 11 panels, each moderated by a panel chair. Reviewers are required to submit preliminary grades for the proposals in their panel a few days before 
the review meeting. During the review meeting, the reviewers meet for three days to discuss, vote, and write final comments about the proposals in their panel. The panel chairs meet for two additional days to discuss, vote, and write final comments about the Medium and Large proposals that have been forwarded to them from the panels. The goal of the panels and the TAC is to provide a ranked list of all of the proposals to the SSC Director.

\subsubsection{Preliminary grades/comments}

Reviewers enter a preliminary grade in SPRITE for each proposal in their panel (see Figure 2). Grades are entered to one decimal place using the full scale: 1 (lowest) -5 (highest), or 0 if the reviewer is conflicted with the proposal. SPRITE automatically calculates each individual reviewer's average grade and standard deviation, as well as the average grade and standard deviation for each proposal. Reviewers can save their grades, logout, and log back in at any time to update their grades until the preliminary grades deadline.

Reviewers may also enter comments and notes about the proposals. The comments will be combined with the comments entered by the other reviewers in their panel into one field per proposal. The notes are separate from the comments, are not viewable by any of the other reviewers, and are for the reviewer's own private use during the panel discussion.

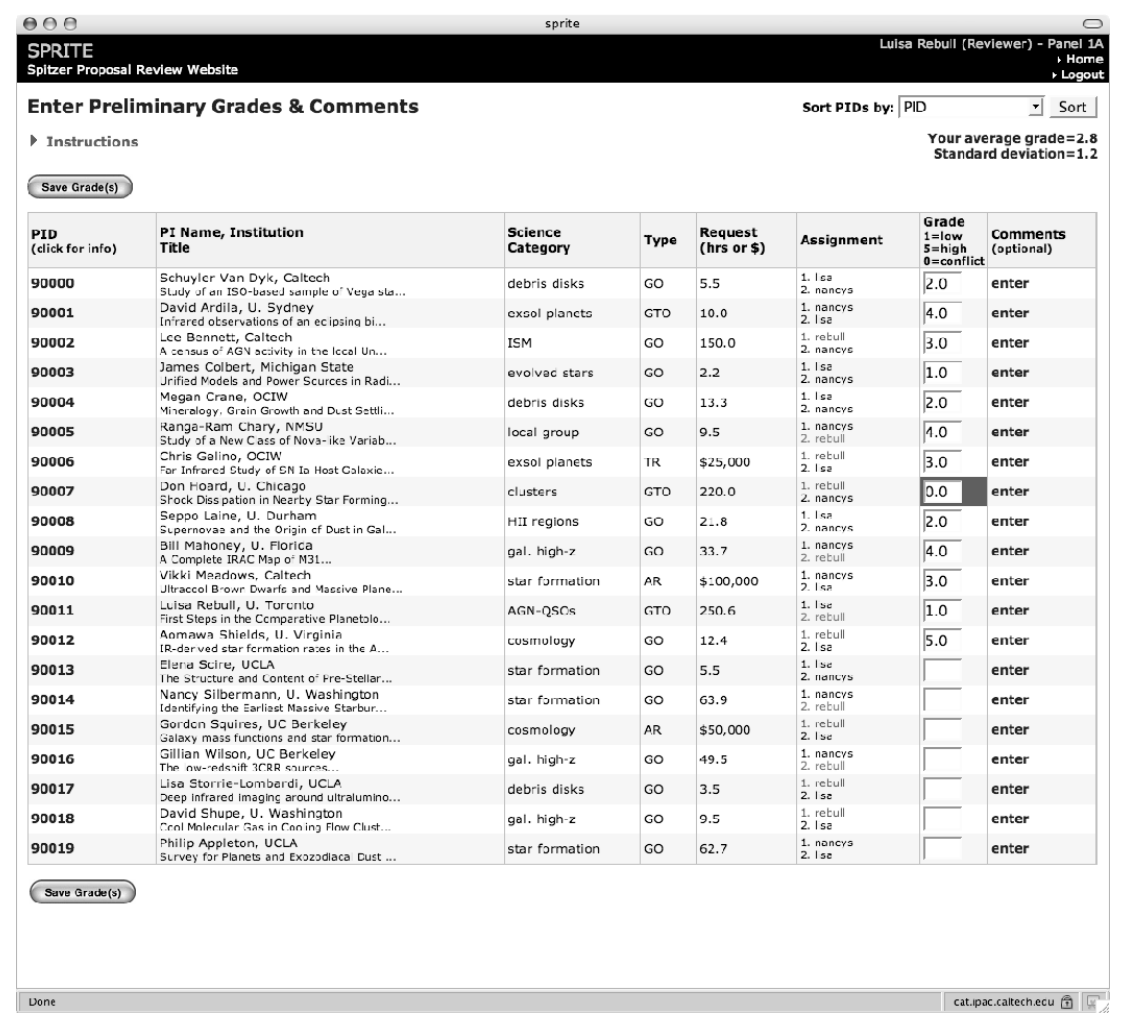

Fig. 2. Reviewers' preliminary grades/comments entry form. Reviewers are able to access the PDF file of the proposal by clicking on the proposal ID number. A pink box indicates a conflict identified by the SSC Community Affairs Team.

\subsubsection{Panel voting/comments}

During the review meeting, reviewers discuss and vote on the proposals in their panel that have not been triaged (see section 3.4.2), using the same grading scale as the preliminary grades. As they vote, the SSC Science User Support Team monitors the voting process (see Section 3.3.1). The primary reviewer of each proposal is responsible for editing the final version of the comments that will be sent to the proposers. The deputy chair and panel chair review and edit all of the comments as needed, producing a final version that will be included in the award letter to the proposer.

\subsubsection{TAC voting}

The panel chairs and TAC chair meet for two additional days to discuss and vote on the Medium and Large proposals that have been forwarded to them from the panels. They also finalize the amount of money allocated to Archival 
Research and Theoretical Research proposals, and finalize all of the comments. The TAC provides a final ranked list of all of the proposals to the SSC Director.

\subsection{SPRITE options for SSC Science User Support Team (SUST)}

SUST members provide technical support for the panel to which they are assigned. This includes answering any questions the panel might have, monitoring the voting process, and generating a ranked list of the proposals in their panel based on the panel's recommendations.

\subsubsection{Monitoring voting}

The SUST members monitor the voting of their panels by making sure each reviewer enters a vote for each proposal in SPRITE. As the reviewers vote, the SUST members must reload their monitoring page (see Figure 3) to see the individual votes show up in the table. SUST members must alert their panel about potential conflicts, which are identified in SPRITE. Reviewers enter 0 for proposals with which they are conflicted, so that SUST members knows that they are truly conflicted and have not just forgotten to enter a vote. As soon as all of the votes have been entered, SUST members enter the voted allocation (hours for Small observing proposals or dollars for Archival and Theoretical proposals). SPRITE calculates the average vote and standard deviation for the proposal, which will then be displayed on the reviewers' voting page. The SUST members may also enter notes about the proposal, which are not visible to the reviewers, including which reviewers were conflicted, any allocation changes, etc.

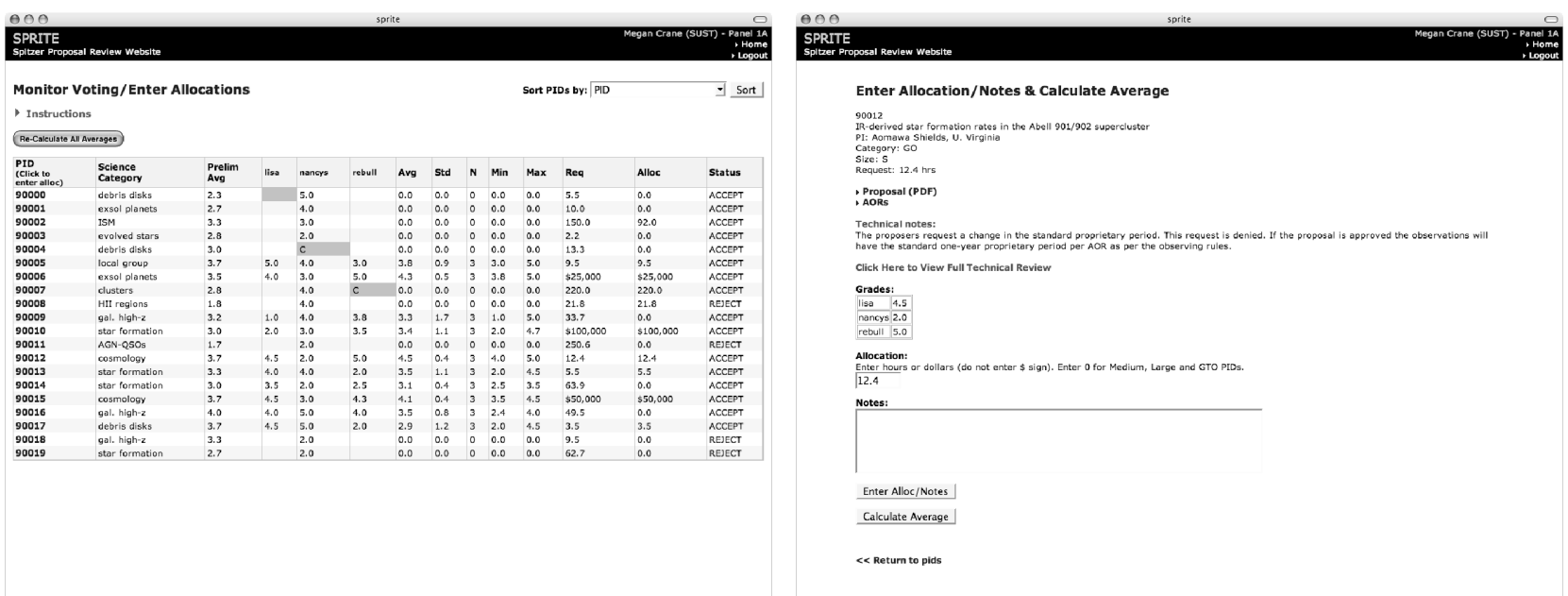

Fig. 3. SUST voting monitoring pages. Left: List of all proposals and each reviewer's individual vote. Conflicts are indicated by pink boxes. Right: Allocation/Notes entry form for one proposal. SUST members can access information about the proposal including the proposal PDF file and any technical information.

\subsubsection{Ranking}

Once the reviewers have voted on all of the non-triaged proposals in their panel, SPRITE assigns an initial rank to the proposals based on the average vote and standard deviation (if two proposals have the same average vote, the one with the lowest standard deviation will be ranked higher). The ranking can then be adjusted based on the panel's recommendation. For example, if one proposal has a lower average vote than another, but the panel wants to rank it higher, the SUST member can enter a higher rank for that proposal, and SPRITE will re-rank the list accordingly. The list of proposals can be sorted by different options, such as the rank, average vote, science category, etc. The cumulative number of voted hours for Small proposals compared to the panel's provisional allocation determines the status (ACCEPT or REJECT) of the proposals (see Figure 4). The full provisional allocation and $50 \%$ and $25 \%$ of the provisional allocation are used to determine the scheduling priority of the proposal. The panel finalizes the status of each Small proposal in their panel, and recommends certain Medium and Large proposals to the TAC by setting the status to PENDING. 


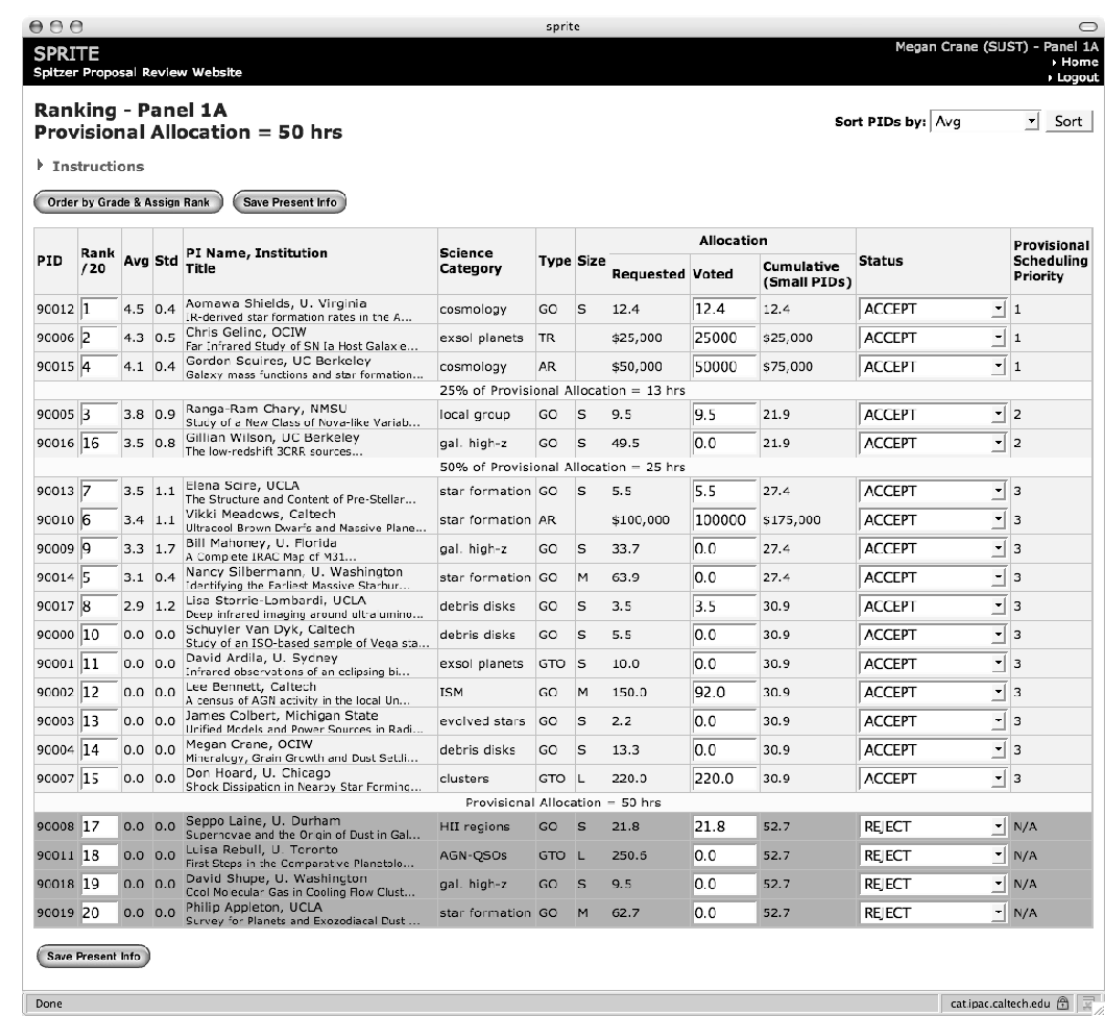

Fig. 4. SUST ranking page. SUST members adjust the rank, voted allocation, and status of the proposals based on the panel's recommendation.

\subsection{SPRITE options for SSC Community Affairs Team (CAT)}

CAT members invite reviewers, assign them to appropriate panels, determine the provisional allocation for each panel, assign proposals to panels, assign primary and secondary reviewers to proposals, identify potential reviewer-proposal conflicts, enter technical notes about the proposals, complete the triage process just prior to the review meeting, support the reviewers and SUST members during the review meeting, monitor the TAC voting and ranking process, review all of the final comments written by the reviewers, and send award letters to the proposers after the review meeting.

\subsubsection{Entering proposal assignments and conflicts}

Proposal assignments (primary and secondary reviewers) and potential conflicts of interest are identified by separate SSC software and loaded into the SPRITE database prior to the review meeting. Assignments and conflicts can then easily be updated via SPRITE, and they are visible both to SUST members and to the reviewers.

\subsubsection{Triage}

Proposals with the lowest preliminary average grades are triaged by CAT members on the Thursday prior to the review meeting. Typically the bottom third of the proposals will be triaged, leaving the panel with a more manageable number to discuss during their meeting. The panels briefly review the triaged proposals at the beginning of their meeting, particularly if there are any that have a large standard deviation in the preliminary average grade, and may elect to pull any out of triage and discuss them during their meeting.

\subsubsection{Monitoring TAC voting \& ranking}

CAT members monitor the TAC voting in the same manner that SUST members monitor the panel voting. CAT members ensure that each reviewer enters a vote for the Medium and Large proposals in the TAC, enter the voted allocations, and calculate the average vote for the proposals. Once the TAC has voted on all of the proposals, SPRITE assigns an initial rank to the proposals based on the average vote, and then CAT members can adjust the rank based on the TAC's recommendation. A final ranked list of all the proposals is generated and forwarded to the SSC Director. 


\subsubsection{Sending award letters}

The SPRITE software is used to send acceptance and rejection letters to the Principal Investigator and co-investigators of all of the proposals after the review meeting, utilizing the PHP mail() function. The comments entered by the reviewers are edited if necessary by CAT members before being sent to the proposers. The SPRITE software is used to send about 700-800 letters to about 5000 recipients. This typically takes about 2-3 hours, and happens a few weeks after the review meeting. The PHP sleep() function is used to impose a 5-second delay between the delivery of letters so that they do not pile up in the SSC mail queue. The letters are usually sent in batches of 20-30 to avoid browser timeout.

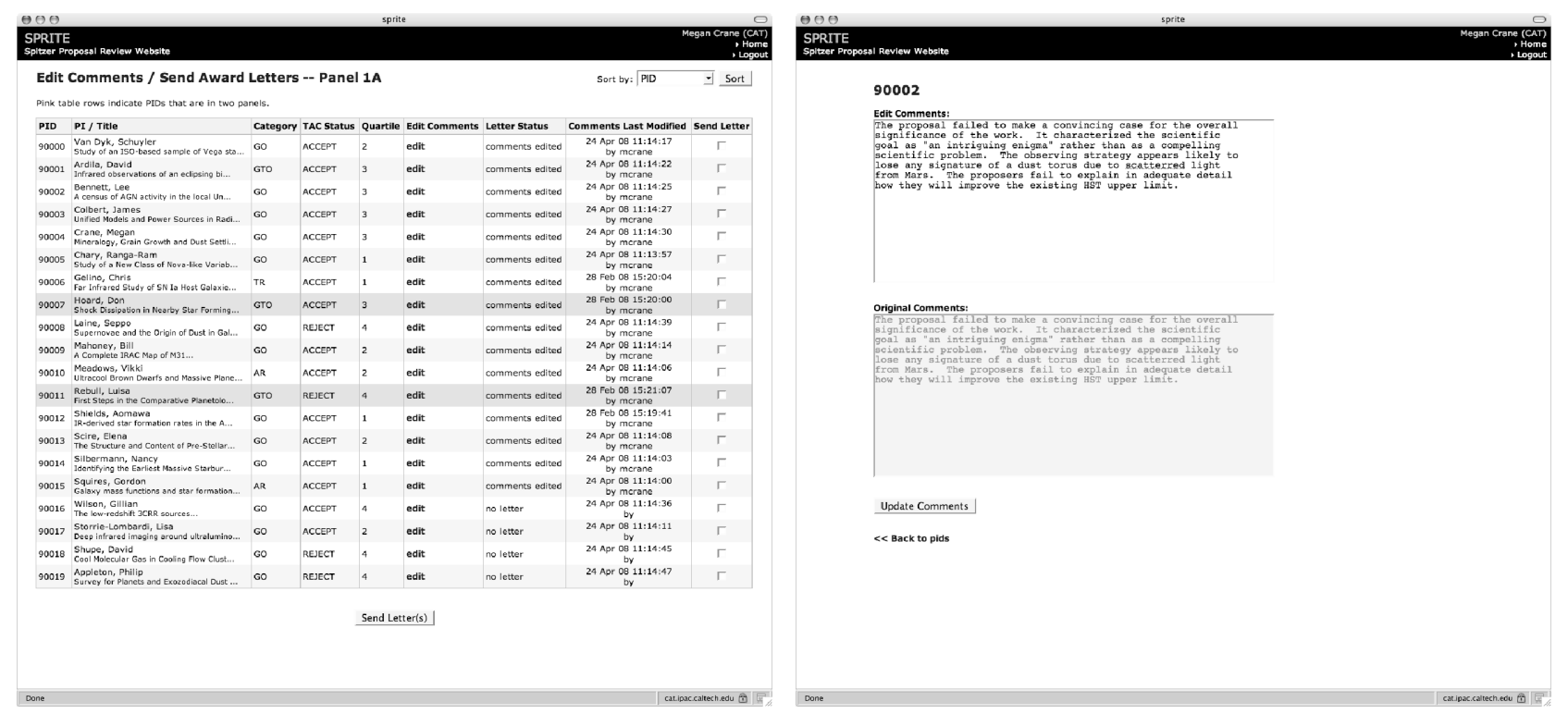

Fig. 5. CAT page for editing reviewer comments and sending award letters. Left: list of all proposals in one panel. Right: comment editing form for one proposal.

\section{LESSONS LEARNED}

SPRITE has been well received by reviewers and SUST members, especially because it eliminates the process of voting by secret ballot (see Section 1.3). Allowing the reviewers to enter their votes electronically eases the workload on the SUST member and allows the reviewers to see their own individual average vote and standard deviation calculated automatically as they vote. Giving SUST members electronic access to the same information that the reviewers see (e.g. technical reviews) makes it easier for them to assist their panel.

PHP has proven to be an exceptional tool in the development of SPRITE for several reasons. Because of its ability to generate PDF files using the free FPDF class (http://www.fpdf.org/), individual instruction sheets for each reviewer can be generated depending on their status (reviewer, panel chair, deputy chair, or TAC chair), which contain their username and password. It is also possible to generate Excel files using PHP, so CAT members can download in Excel format the preliminary grades as well as the final ranked list of proposals for each panel. Finally, the ability to send award letters automatically using the PHP mail() function saves an enormous amount of time and energy.

The process by which reviewers enter comments about the proposals continues to be problematic. The first year SPRITE was implemented, only the primary reviewer had permission to edit the final comments before the panel chair would approve them. The reviewers complained that at least both the primary and secondary reviewers should be able to edit the comments, if not all of the reviewers in the panel. The second year SPRITE was used, all of the reviewers in the panel were given access to edit the final comments. This resulted in some reviewers overwriting each other's comments if they did not communicate to each other who was editing the comments. The SSC will continue to investigate the best process to use.

PHP session management includes a built-in method for "garbage collection" which means that it will destroy a user's session if the user is idle for a specified amount of time. The default PHP value is 1440 seconds, or 24 minutes. Since the time that the reviewers spend discussing proposals before entering a vote often lasts longer than 24 minutes, this 
value had to be increased to 3 hours to ensure that users did not get unintentionally logged out. This was achieved by setting the PHP directive session.gc_maxlifetime to 10800 and rebooting the webserver.

Since SPRITE is web-based software, a strong and reliable Internet connection is essential. If the Internet connection is unstable, session information can be lost, and reviewers are at risk of losing some or all of their grades. In this situation, the only solution is to move back to the process of voting non-electronically by secret ballot with SUST entering the grades into a spreadsheet by hand.

\section{REFERENCES}

[1] Legassie, M. S., Bennett, L., Comeau, S. B., and Dodd, S. R., "Advanced load-testing techniques for a science archive", in Observatory Operations: Strategies, Processes, and Systems II, edited by Roger J. Brissenden, David R. Silva, Proceedings of SPIE Vol. 7016 (SPIE, Bellingham, WA, 2008), In Press.

[2] Laine, S., Silbermann, N. A., Rebull, L. M., and Storrie-Lombardi, L. J., "Spitzer Space Telescope proposal process," Proc. SPIE 6270, 627029 (2006). 